\title{
Complex Geometric Structure of a Simple Solid-Liquid Interface: GaN(0001)-Ga
}

\author{
A. E. F. de Jong $\odot,{ }^{1,2}$ V. Vonk $\odot,{ }^{3, *}$ M. Boćkowski®${ }^{4}$ I. Grzegory $\odot,{ }^{4}$ V. Honkimäki®, ${ }^{2}$ and E. Vlieg $\oplus^{1, \dagger}$ \\ ${ }^{1}$ Radboud University, Institute for Molecules and Materials, Heyendaalseweg 135, 6525AJ Nijmegen, Netherlands \\ ${ }^{2}$ European Synchrotron Radiation Facility, CS 40220, F-38043, Grenoble Cedex 9, France \\ ${ }^{3}$ DESY NanoLaboratory, Deutsches Elektronen-Synchrotron (DESY), Notkestraße 85, D-22607 Hamburg, Germany \\ ${ }^{4}$ Institute of High Pressure Physics PAS, Sokolowska 29/37, 01-142 Warsaw, Poland
}

(Received 16 August 2019; accepted 29 January 2020; published 24 February 2020)

\begin{abstract}
The equilibrium atomic interface structure between $\mathrm{Ga}$ and $\mathrm{GaN}(0001)$ is shown to contain substrate surface vacancies followed by substrate-induced layering and preferential lateral ordering in the liquid. The uncovered presence of point defects, in the form of vacancies at both sides of the solid-liquid interface, is an important structural feature which governs the local physical properties. Our x-ray diffraction study reveals that the layering is very stable and persists up to a temperature of $1123 \mathrm{~K}$ and a nitrogen pressure of $32 \mathrm{bar}$. The Ga layer spacing agrees remarkably well with the Friedel oscillation period for this system.
\end{abstract}

DOI: 10.1103/PhysRevLett.124.086101

Solid-liquid interfaces are omnipresent in everyday life and are encountered in many industrial applications. Although studied for quite some time, a lack of detailed atomic-scale descriptions of these interfaces hampers the level of understanding needed to predict and control such processes as crystal growth from solution, soldering, and friction. Metal-semiconductor interfaces have proven particularly useful model systems for experimental $[1,2]$ and theoretical [3] studies of solid-liquid interfaces. Compared with, for example, water, monatomic metals with a low melting temperature have a more simple theoretical description and, in general, are easier to interpret experimentally. Such systems are also relevant in semiconductor applications.

Gallium nitride has become the most important semiconductor material after silicon. The GaN band gap of about $3.4 \mathrm{eV}$ enables a range of applications, most prominently blue light-emitting diodes and power electronics [4,5]. Although vapor phase epitaxy [6] is the most widespread $\mathrm{GaN}$ growth technique, the highest-quality crystals are presently obtained by the ammonothermal growth method [7], which takes place at a high ammonia pressure $(2 \mathrm{kbar})$ and temperature $(1000 \mathrm{~K})$. The growth interface common to all $\mathrm{GaN}$ solution growth techniques is that of liquid $\mathrm{Ga}$ in contact with solid $\mathrm{GaN}$ at a high temperature $(T>1000 \mathrm{~K})$ and a high nitrogen or ammonia pressure $(P>5$ bar). They mostly differ in the way nitrogen is introduced, because there is a large energy barrier for the dissociation and dissolution of this species. We present the temperature and nitrogen pressure dependence of the GaN-Ga solid-liquid interface structure using the Ga-terminated (0001) surface. The $(P, T)$ range used in this study brings the interface near equilibrium under conditions where very slow crystal growth can take place [8]. This allows one to obtain a detailed atomic-scale resolution picture of the structure by using high-energy $\mathrm{x}$-ray diffraction in a grazing-incidence-transmission geometry, which is needed to reach the deeply buried solid-liquid interface [9]. We use this scheme, together with a highpressure furnace [10], to obtain extensive surface crystallographic datasets containing crystal truncation rods (CTRs) [11-13]. High-quality epiready ammonothermal GaN (0001) substrates (Unipress, Poland), having a wurtzite structure with cell parameters $a=3.190$ and $c=5.189 \AA$, were mechanochemically polished, resulting in a surface roughness below $2 \AA$. Our sample furnace was carefully cleaned, and loading of the substrate and liquid $\mathrm{Ga}$ was done inside a glove box to prevent exposure to water and oxygen. The experiment was performed at beam line ID15A of the European Synchrotron Radiation Facility (ESRF). More experimental details can be found elsewhere $[8,10]$. An empirically found recipe, consisting of heating under a controlled nitrogen atmosphere, was used to optimally contact the liquid gallium with the substrate [8]. This procedure repairs the surface and cleans residual impurities [14], resulting in a well-defined and atomically smooth interface at near-equilibrium conditions at the nitrogen pressures used.

The experiment was performed using an x-ray energy of $71 \mathrm{keV}$ in combination with a hybrid pixel detector equipped with a CdTe sensor [15]. The CTR intensities were collected in the so-called stationary mode and corrected for scattering geometry factors, such as transmission through the chamber wall, Ga liquid, and its container, to obtain structure factors [16].

The most extensive dataset was recorded at a temperature of $473 \mathrm{~K}$ and 11 bar of nitrogen and consists of four unique rods (see Fig. 1). These are reduced from a larger dataset by averaging over symmetry equivalents (space group $\mathrm{Pmm}$ ), which resulted in a multiplicity of 2.2 and an agreement 

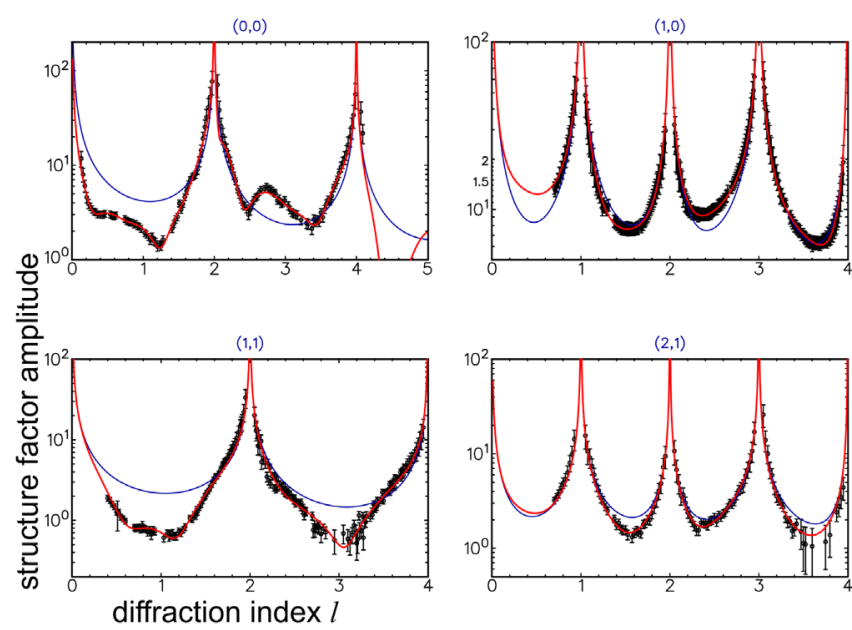

FIG. 1. Data and fit obtained at $473 \mathrm{~K}$ and 11 bar of $\mathrm{N}_{2}$ pressure. Data are represented as circles plus error bars, fit as red curves, and bulk contribution as blue curves. The enlarged error bars on the $(2,1)$ rod around $l=3.5$ are caused by the very weak signal.

factor of $8.2 \%$. The data differ considerably from those for bulk-terminated GaN (blue curves), directly showing the ordering of the interfacial Ga. Figure 2 shows the structural model used to fit the data [17]. A wide range of models was tested with different lateral positions and varying degrees of lateral and perpendicular ordering (as modeled through anisotropic Debye-Waller parameters [18]). The model presented here is the simplest that accurately fits the data. Four layers are found to be present with both lateral and perpendicular order (Ga1-Ga4), while subsequent layers have only a rapidly decreasing perpendicular order (layering). Beyond layer Ga6 on, the so-called distorted crystal model [19] is used, yielding a smooth transition to the bulk liquid density of Ga. The model includes relaxations and partial occupancies in the topmost $\mathrm{GaN}$ layer and liquid $\mathrm{Ga}$ layers. The occupancies are expressed in monolayers (ML), where $1 \mathrm{ML}$ is one atom per surface unit cell.

The optimum fitting parameters are listed in Table I and yield a $\chi^{2}$ value of 0.66 . The most important distances between $\mathrm{Ga}$ atoms as derived from our fit are shown in Fig. 2. The Ga0-Gal distance of $2.24 \AA$ is somewhat shorter than the sum of the atomic radii $(2.45 \AA)$. The formation of $\mathrm{Ga}$ dimers in liquid $\mathrm{Ga}$ has often been discussed [20-22]. Here, the distance around $2.86 \AA$ of $\mathrm{Ga} 0-\mathrm{Ga} 3$ and $\mathrm{Ga} 1-\mathrm{Ga} 4$ is consistent with such dimers. We find that the surface has no significant roughness, confirming the equilibrium conditions at the interface. A striking feature is the presence of $20 \%$ vacancies in the topmost Ga layer (Ga0) of the crystal. The next layer (Ga1) is a liquid with strong ordering properties and a location above the hollow site (H3). Instead of the expected occupancy around $1 \mathrm{ML}$, this layer has an occupancy of only $0.35 \mathrm{ML}$. The remaining hollow sites are partly occupied by a layer that is located at a larger distance



FIG. 2. A side view of the (0001) GaN-Ga interface with the size of the atoms corresponding to the amount of disorder. Layers $\mathrm{Ga} 5$ and Ga6 have no in-plane order, and the color of the atoms $\mathrm{Ga} 2, \mathrm{Ga} 3$, and $\mathrm{Ga} 4$ is lighter to highlight their fractional occupancies. Interatomic distances are given in angstroms.

(Ga2). Ga2 is part of a layer that contains also atoms located at $T 1(\mathrm{Ga} 4)$ and $T 4(\mathrm{Ga} 5)$ sites and with a total occupancy of 1.3 ML. This layer shows significant disorder, and, thus, the atoms are quite mobile and will diffuse from site to site. The occupancies therefore correspond to effective residence fractions per site. As drawn in Fig. 2, the $\mathrm{Ga} 3, \mathrm{Ga} 4$, and $\mathrm{Ga} 5$ atoms are very close together, but the partial occupancy means there is enough room. Subsequent layers are laterally disordered $\left(B_{\|}=\infty\right)$. The complete density profile is shown in Fig. 3. The analysis is most sensitive to the first layers. For example, not having the lowest liquid atom in the hollow site nearly doubles the $\chi^{2}$ value, while fixing the occupancy of $\mathrm{Ga} 0$ to 1 gives a $\chi^{2}$ of 1.05 .

In addition to the data at $473 \mathrm{~K}$ and $11 \mathrm{bar},(00)$ and (10) rods were measured at the following temperature-pressure combinations: $293 \mathrm{~K}-11$ bar, $873 \mathrm{~K}-13$ bar, $873 \mathrm{~K}-27$ bar, and $1123 \mathrm{~K}-32$ bar. We found that these more limited datasets did not allow extraction of full structural details,

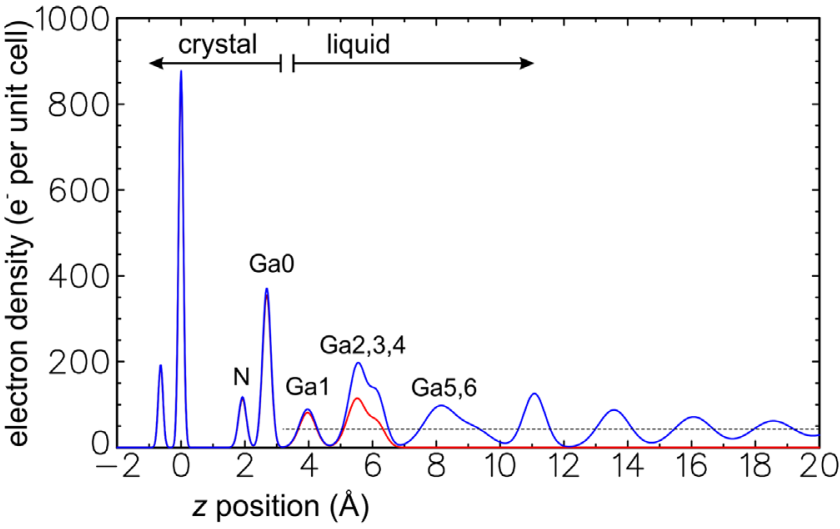

FIG. 3. Projected electron density along the interface normal $(z$ direction) obtained from the best fit model. The blue curve is the full density; the red one shows the $(1,0)$ Fourier component, i.e., only the density with in-plane order. 


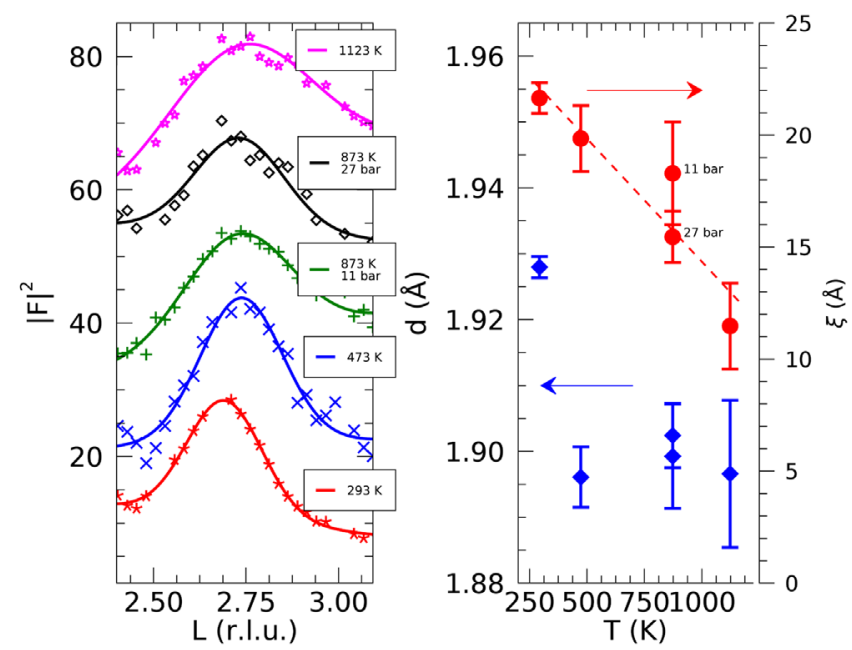

FIG. 4. (Left) Quasi-Bragg peaks due to Ga layering at different temperatures and pressures. Shown are experimental data points and Gaussian fits (lines). (Right) Average layer spacing and correlation length $(\xi)$ deduced from the peak positions and width of the quasi-Bragg peaks as a function of the temperature.

and merely the quasi-Bragg peaks were analyzed. This peak, which appears on the specular rod around $l=2.7$, arises from constructive interference between layers of $\mathrm{Ga}$ atoms. The corresponding $d$ spacing of approximately $1.9 \AA$ represents the weighted average of interplanar distances as probed by the $\mathrm{x}$ rays. Therefore, the appearance of the quasi-Bragg peak is direct evidence for the layering, and its position and width can be used to obtain a picture of the layer spacing $(d=c / l)$ and correlation length $(\xi=c / \Delta l)$, respectively. The relevant data, together with the extracted $d$ spacing and correlation length, are shown in Fig. 4.

The $d$ spacing decreases when heating from 293 to $473 \mathrm{~K}$ and then does not change significantly anymore over the investigated temperature-pressure range. At $293 \mathrm{~K}$, which is just below the Ga freezing temperature of $302.9 \mathrm{~K}$, we did not observe any Bragg reflections from its solid phase, and we conclude that it was in a supercooled state.
Interestingly, the drop in $d$ spacing of about $1 \%$ is in line with gallium's density increase when melting. In the temperature range $473-1223 \mathrm{~K}$, the $d$ spacing is found to be constant and not increasing by the $5 \%$ as expected from thermal expansion [24]. This value is way beyond the determined error bar. It could be that the substrate-induced lateral interaction, which correlates with the liquid layer spacing, annihilates its increase due to Ga thermal expansion [25]. The correlation length of the layering decreases approximately linearly with temperature; see Fig. 4. Thermal vibration can explain this, since its squared amplitude scales linearly with the temperature to first order in the harmonic approximation [26]. The correlation length of approximately $20 \AA$ near room temperature has also been found for other solid-liquid metal-semiconductor interfaces $[25,27,28]$. The linear decrease of the correlation with an increasing temperature has been observed for room-temperature ionic liquids (RTILs) in contact with a hard wall as well [29]. It is quite remarkable that here it persists to temperatures above $1000 \mathrm{~K}$. If the trend would continue, it is expected that around a temperature of $1500 \mathrm{~K}$, where the value will drop below $5 \AA$ (about two atomic layers), the layering will disappear. Both RTILs and gallium are known for their broad liquidus range, and it seems that, by extrapolation of the correlation lengths, the layering at a hard wall could persist up to roughly halfway to their boiling points. The pressures studied here are approximately 2 orders of magnitude lower than those for which pressure effects are expected [30].

There are no directly comparable data on the interface we investigated, but the structure of several MLs of Ga under vacuum conditions on $\mathrm{GaN}(0001)$ has been studied $[31,32]$. It was found that a dense Ga bilayer can form, of which the layer in direct contact with the $\mathrm{GaN}$ has a coverage of 1 and the second of 4/3 ML; hence, it was coined the contracted bilayer. This particular model (and ones like it) strongly disagrees with our data (and model) for the conditions with a thick Ga layer. First of all, in a vacuum the first layer was reported to have a coverage of $1 \mathrm{ML}$, a $T 1$ position, and a distance of $2.5 \AA$ to the

TABLE I. Fit results. Optimum parameter values for the fit at $473 \mathrm{~K}$ and 11 bar $\mathrm{N}_{2}$. The parameters $B_{\perp}$ and $B_{\|}$ indicate the out-of-plane and in-plane, respectively, Debye-Waller factors of which the bulk values are taken from Ref. [23]. Parameters that are nonrefined are listed without an error bar.

\begin{tabular}{lccccc}
\hline \hline Atom & Location & $z(\AA)$ & Occupancy $(\mathrm{ML})$ & $B_{\|}\left(\AA^{2}\right)$ & $B_{\perp}\left(\AA^{2}\right)$ \\
\hline $\mathrm{Ga}$ & Layered & $11.1 \pm 0.1$ & & & \\
$\mathrm{Ga} 6$ & & $9.1 \pm 0.2$ & $0.3 \pm 0.1$ & $\infty$ & $20 \pm 5$ \\
$\mathrm{Ga} 5$ & & $8.1 \pm 0.1$ & $0.7 \pm 0.2$ & $\infty$ & $19 \pm 4$ \\
$\mathrm{Ga} 4$ & $T 4$ & $5.1 \pm 0.1$ & $0.5 \pm 0.1$ & $25 \pm 5$ & $5 \pm 2$ \\
$\mathrm{Ga} 3$ & $T 1$ & $5.47 \pm 0.1$ & $0.35 \pm 0.1$ & $40 \pm 20$ & $4 \pm 2$ \\
$\mathrm{Ga} 2$ & $H$ & $3.97 \pm 0.02$ & $0.50 \pm 0.05$ & $5 \pm 1$ & $7 \pm 2$ \\
$\mathrm{Ga} 1$ & $H$ & $2.69 \pm 0.01(\Delta=0.09)$ & $0.36 \pm 0.03$ & $3.0 \pm 0.3$ & $5 \pm 2$ \\
$\mathrm{Ga} 0$ & & $1.96 \pm 0.02(\Delta=-0.03)$ & 1 & $1.3 \pm 0.2$ & $1.4 \pm 0.3$ \\
$\mathrm{~N}$ & & & $1.2 \pm 0.3$ & \\
\hline \hline
\end{tabular}


substrate, while we find (for Ga1) 0.36 ML, a hollow site, and $1.3 \AA$, respectively. The spacing to the second layer was found to be $2.4 \AA$ in a vacuum, while we obtain $1.8 \AA$. Nevertheless, the general structural features of the two models are similar, namely, that of a first layer in registry with the underlying substrate and a second layer with $\sim 4 / 3$ ML density and variations in the lateral registry. We thus find a much smaller layer spacing of the first two layers compared to the few-ML vacuum case. A theoretical study of the Ga-thickness-dependent (electronic) structure of the $\mathrm{Ga}-\mathrm{GaN}(0001)$ interface indicates that there is a transition around $5 \mathrm{ML}$ towards a true metal-semiconductor contact, at which point electron transfer takes place [33]. It has been hypothesized that such an electron transfer mechanism may be responsible for a rather exotic quasiliquid structure which manifests itself in enhanced local density and shortened bond lengths [27]. Note that, in contrast to our finding, the theoretical study assumed adsorption on the $T 1$ location and gave a spacing to the topmost Ga layer of $1.75 \AA$. At the very interface, i.e., the last crystal layer $(\mathrm{Ga} 0)$ and the first quasiliquid transition layer (Ga1), we find an appreciable number of atomic vacancies, which lead to a density deficit compared to a bulk terminated situation. Indications of atomic vacancies in the two layers adjacent to the solid-liquid interface have been observed before [18,25,34], but the quality of the interface and/or x-ray scattering power of the systems in those studies did not allow for any solid conclusions to be drawn. In the present case, the high quality of the system gives a high confidence in the presence of these defects. Atomic vacancies in the transition layer have been discussed to arise due to the negligible chemical potential difference between the liquid and this layer; the energy for vacancy formation depends on an interfacial dipole, which is created by electron transfer [35]. In the case of the $\mathrm{Ga}-\mathrm{GaN}$ interface, the interfacial dipole is influenced by the solid's polar (0001) surface [36], which complicates a simple description, but may play a role in the formation of vacancies in the last layer of the crystal. Alternatively, the vacancies could indicate a surface reconstruction, but this is unlikely in view of the earlier results on the thin layers in a vacuum [31,32].

The crystallographic description of the geometric structure presented above gives a detailed picture of the atomic positions. From a continuum, electronic point of view, the layering and its period can be described through so-called Friedel oscillations $[37,38]$. An oscillatory term $\cos \left(2 k_{F} r\right)$, with $k_{F}$ the Fermi wave vector and $r$ a distance, leads to density oscillations with periodicity $\lambda_{\mathrm{Fr}}=\pi / k_{F}$. Damping of this potential depends on the dimensionality and resulting Fermi surface shape [39], and bulk liquid Ga can be described using an additional $1 / r^{3}$ term [40]. The fact that the observed layering persists up to several nanometers from the interface suggests that the damping of the 2D quasiliquid falls off less rapidly than in the bulk, like $1 / r^{d}$, with $d<3$ [39].
TABLE II. Comparison between experimentally obtained layer spacings and Friedel oscillation periods for different metals [41].

\begin{tabular}{lcc}
\hline \hline Metal & $d_{\text {exp }}(\AA)$ & $\lambda_{\mathrm{Fr}}(\AA)$ \\
\hline $\mathrm{Ga}$ & 1.9 (this study) & 1.9 \\
$\mathrm{In}$ & $2.2[25]$ & 2.1 \\
$\mathrm{Au}_{0.6} \mathrm{In}_{0.4}$ & $2.3[45]$ & $2.4^{\mathrm{a}}$ \\
\hline
\end{tabular}

${ }^{\mathrm{a}} \mathrm{A}$ weighted average of the two pure metals is taken.

Interestingly, the values of $\lambda_{\mathrm{Fr}}$ for different metals are determined only by the occupation of the energy levels, and these have been tabulated [41]. Table II lists these values together with experimental spacings obtained from x-ray diffraction experiments that showed a clear quasi-Bragg peak, like the study presented here. We find a very good agreement between the experiment and theory, unlike the liquid $\mathrm{Ga}$ spacing obtained from another experimental study, where no strong quasi-Bragg peak was observed [42]. Finally, the values obtained for the liquid layer spacing at solid liquid interfaces are considerably smaller than those found at the free surfaces of liquid Ga and In of 2.5 [43] and $2.7 \AA$ [44], respectively. This may point to a different electronic structure between the buried and free interfaces. It is unclear where these discrepancies and differences may come from and invites theorists to develop a sophisticated model.

In conclusion, the preparation of an equilibrated $\mathrm{Ga}-\mathrm{GaN}$ (0001) interface allows its reliable and detailed structure determination. The overall structure is simple, going smoothly from a partly ordered layer to a bulk Ga liquid. Surprising, however, are the high density of Ga vacancies in the topmost GaN layer, the low density in the first Ga layer, and the high density of the second $\mathrm{Ga}$ layer. Also unexpected is the persistence of the layering up to a temperature of $1123 \mathrm{~K}$ and a nitrogen pressure of 32 bar. The experimental results differ considerably from those obtained on the same interface but with only a few $\mathrm{Ga}$ layers and in a vacuum. The layering distance found here compares well with the expected Ga Friedel oscillation period, which arises due to screening effects and may be a general feature. Such electronic effects and the presence of point defects at solid-liquid interfaces are important in view of a full understanding of electron and ion transport at solid-liquid interfaces.

We like to acknowledge financial support received from the ESRF and the Netherlands Organization for Scientific Research (NWO) through a VENI grant.

* Corresponding author.

vedran.vonk@desy.de

Corresponding author.

e.vlieg@science.ru.nl

[1] H. Reichert, O. Klein, H. Dosch, M. Denk, V. Honkimäki, T. Lippmann, and G. Reiter, Nature (London) 408, 839 (2000). 
[2] L. Tamam, D. Pontoni, T. Hofmann, B. M. Ocko, H. Reichert, and M. Deutsch, J. Phys. Chem. Lett. 1, 1041 (2010).

[3] M. Zhao and S. A. Rice, J. Phys. Chem. A 115, 3859 (2011).

[4] P. Pust, P. Smidt, and W. Schnick, Nat. Mater. 14, 454 (2015).

[5] J. Milllan, P. Godignon, X. Perpina, A. Perez-Tomas, and J. Rebollo, IEEE Trans. Power Electron. 29, 2155 (2014).

[6] T. Yao and S.-K. Hong, Oxide and Nitride Semiconductors: Processing, Properties, and Applications (Springer Science \& Business, New York, 2009), Vol. 12.

[7] M. Zajac, R. Kucharski, K. Grabianska, A. GwardysBak, A. Puchalski, D. Wasik, E. Litwin-Staszewska, R. Piotrzkowski, J. Domagala, and M. Bockowski, Prog. Cryst. Growth Charact. Mater. 64, 63 (2018).

[8] A. E. F. de Jong, V. Vonk, G. Kamler, M. Boćkowski, I. Grzegory, V. Honkimäki, and E. Vlieg, J. Cryst. Growth 448, 70 (2016).

[9] H. Reichert, V. Honkimäki, A. Snigirev, S. Engemann, and H. Dosch, Physica (Amsterdam) 336B, 46 (2003).

[10] A. E. F. de Jong, V. Vonk, V. Honkimäki, B. Gorges, H. Vitoux, and E. Vlieg, J. Cryst. Growth 420, 84 (2015).

[11] M. Von Laue, Ann. Phys. (N.Y.) 418, 55 (1936).

[12] I. K. Robinson, Phys. Rev. B 33, 3830 (1986).

[13] E. Vlieg, in Surface and Interface Science (Wiley-VCH, New York, 2012), pp. 375-425.

[14] V. M. Bermudez, Surf. Sci. Rep. 72, 147 (2017).

[15] M. Ruat and C. Ponchut, IEEE Trans. Nucl. Sci. 59, 2392 (2012).

[16] E. Vlieg, J. Appl. Crystallogr. 30, 532 (1997).

[17] E. Vlieg, J. Appl. Crystallogr. 33, 401 (2000).

[18] M. F. Reedijk, J. Arsic, F. F. A. Hollander, S. A. de Vries, and E. Vlieg, Phys. Rev. Lett. 90, 066103 (2003).

[19] O. M. Magnussen, B. M. Ocko, M. J. Regan, K. Penanen, P. S. Pershan, and M. Deutsch, Phys. Rev. Lett. 74, 4444 (1995).

[20] T. Yu, J. Chen, L. Ehm, S. Huang, Q. Guo, S.-N. Luo, and J. Parise, J. Appl. Phys. 111, 112629 (2012).

[21] R. Tonner and N. Gaston, Phys. Chem. Chem. Phys. 16, 24244 (2014).

[22] L. Comez, A. Di Cicco, M. Minicucci, R. Tossici, J. Itie, and A. Polian, J. Synchrotron Radiat. 8, 776 (2001).

[23] M. Schowalter, A. Rosenauer, J. T. Titantah, and D. Lamoen, Acta Crystallogr. Sect. A 65, 227 (2009).

[24] M. J. Assael, I. J. Armyra, J. Brillo, S. V. Stankus, J. Wu, and W. A. Wakeham, J. Phys. Chem. Ref. Data 41, 033101 (2012).

[25] V. Vonk, M. Cremers, A. de Jong, S. Pintea, and E. Vlieg, Surf. Sci. 621, 69 (2014).
[26] B. E. Warren, X-Ray Diffraction (Dover, New York, 1969).

[27] H. Reichert, M. Denk, J. Okasinski, V. Honkimäki, and H. Dosch, Phys. Rev. Lett. 98, 116101 (2007).

[28] V. Vonk, D. Pontoni, M. Cremers, A. Kerkenaar, A. A. C. Bode, W. Szweryn, G. Nowak, A. E. F. de Jong, H. Dosch, and E. Vlieg, Langmuir 33, 814 (2017).

[29] M. Mezger, H. Schröder, H. Reichert, S. Schramm, J. S. Okasinski, S. Schoder, V. Honkimäki, M. Deutsch, B. M. Ocko, J. Ralston, M. Rohwerder, M. Stratmann, and H. Dosch, Science 322, 424 (2008).

[30] R. Li, L. Wang, L. Li, T. Yu, H. Zhao, K. Chapman, Y. Wang, M. Rivers, P. Chupas, and H. Liu, Sci. Rep. 7, 5666 (2017).

[31] A. R. Smith, R. M. Feenstra, D. W. Greve, M. S. Shin, M. Skowronski, J. Neugebauer, and J. E. Northrup, J. Vac. Sci. Technol. B 16, 2242 (1998).

[32] J. E. Northrup, J. Neugebauer, R. M. Feenstra, and A. R. Smith, Phys. Rev. B 61, 9932 (2000).

[33] P. Witczak, P. Kempisty, P. Strak, and S. Krukowski, J. Vac. Sci. Technol. A 33, 061101 (2015).

[34] J. Arsic, D. Kaminski, N. Radenovic, P. Poodt, W. Graswinckel, H. Cuppen, and E. Vlieg, J. Chem. Phys. 120, 9720 (2004).

[35] J. Kang, J. Zhu, C. Curtis, D. Blake, G. Glatzmaier, Y.-H. Kim, and S.-H. Wei, Phys. Rev. Lett. 108, 226105 (2012).

[36] P. W. Tasker, J. Phys. C 12, 4977 (1979).

[37] J. Friedel, Philos. Mag. 43, 153 (1952).

[38] B. G. Walker, N. Marzari, and C. Molteni, J. Phys. Condens. Matter 18, L269 (2006).

[39] A. M. Gabovich, L. G. Ilchenko, E. A. Pashitskii, and Y. A. Romanov, Surf. Sci. 94, 179 (1980).

[40] L. Bove, F. Formisano, F. Sacchetti, C. Petrillo, A. Ivanov, B. Dorner, and F. Barocchi, Phys. Rev. B 71, 014207 (2005).

[41] N. W. Ashcroft and N. D. Mermin, Solid State Physics (Brooks-Cole, Belmont, MA, 1976).

[42] W. J. Huisman, J. F. Peters, M. J. Zwanenburg, S. A. de Vries, T. E. Derry, D. Abernathy, and J. F. van der Veen, Nature (London) 390, 379 (1997).

[43] M. J. Regan, E. H. Kawamoto, S. Lee, P. S. Pershan, N. Maskil, M. Deutsch, O. M. Magnussen, B. M. Ocko, and L. E. Berman, Phys. Rev. Lett. 75, 2498 (1995).

[44] H. Tostmann, E. DiMasi, P. S. Pershan, B. M. Ocko, O. G. Shpyrko, and M. Deutsch, Phys. Rev. B 59, 783 (1999).

[45] R. E. Algra, V. Vonk, D. Wermeille, W. J. Szweryn, M. A. Verheijen, W. J. P. van Enckevort, A. A. C. Bode, W. L. Noorduin, E. Tancini, A. E. F. de Jong, E. P. A. M. Bakkers, and E. Vlieg, Nano Lett. 11, 44 (2011). 